\title{
An Experimental Examination of Binge Watching and Narrative Engagement
}

\author{
Sarah E. Erickson ${ }^{1, * \mathbb{D}}$, Sonya Dal Cin ${ }^{2,3}$ and Hannah Byl ${ }^{2}$ \\ 1 Department of Communication, Trinity University, 1 Trinity Place, San Antonio, TX 78212, USA \\ 2 Department of Communication Studies, University of Michigan, North Quadrangle, 105 S. State St., \\ Ann Arbor, MI 48109-1285, USA; sdalcin@umich.edu (S.D.C.); hjbyl@umich.edu (H.B.) \\ 3 Research Center for Group Dynamics, Institute for Social Research, 426 Thompson St, \\ Ann Arbor, MI 48106-1248, USA \\ * Correspondence: serickso@trinity.edu
}

Received: 30 November 2018; Accepted: 7 January 2019; Published: 11 January 2019

\begin{abstract}
Increasingly, audiences are engaging with media narratives through the practice of binge watching. The effects of binge watching are largely unknown, although early research suggests binge watching may be motivated by a need for escape and could be associated with some qualities of addiction. In this study, we ask whether the practice of binge watching impacts audience engagement with a media narrative. Using an experimental approach, we manipulate the format of exposure to media narratives (binge or nonbinge) and test the effect of this manipulation on audience engagement, specifically parasocial relationships with favorite characters and narrative transportation. Results suggest that binge watching increases the strength of parasocial relationships and the intensity of narrative transportation. Media engagement has been shown to increase media effects, suggesting that binge watching could change not only how audiences engage with narrative media but also the effect it has on them.
\end{abstract}

Keywords: binge watching; narrative transportation; parasocial relationships; media engagement; narrative engagement; streaming television

\section{Introduction}

Audiences are consuming more television, at a faster pace, than ever before (Campbell et al. 2012). Alternative platforms, such as Netflix, Amazon, and Hulu, are increasingly positioning themselves as major media producers and distributors. For example, according to Netflix's 2017 annual report, over 117 million people in over 190 countries subscribe to Netflix, watching 140 million hours of TV shows and movies per day (Netflix Annual Report 2017). These alternative platforms are changing the ways in which consumers are engaging with media, particularly television (Feeney 2014). Whereas viewing of a series on broadcast television occurred largely on an episodic (e.g., once-a-week) schedule, streaming platforms provide users with more options for, and control over, viewing schedules, including the sort of multi-episode-in-a-single-session practices that some have termed "binge watching." According to a survey conducted by Harris Interactive (sponsored by Netflix and thus to be taken with some skepticism), over $61 \%$ of Netflix users report that they binge watch regularly (Harris Interactive 2013). If these were even close to the numbers in 2013, increases in streaming capabilities and availability since that time suggest even higher levels of binge watching today. But what are the implications of these changes in viewing practices for media audiences, particularly the connection that audiences feel to media texts? As this special issue asks, does binge watching engender deeper connections between the viewer and media content? In this study, binge watching is experimentally manipulated to examine its impact on engagement with media narratives, specifically through two common 
indicators of engagement: narrative transportation and parasocial relationships with media characters. Participants are assigned to binge watch or serially (one episode weekly) watch an assigned unfamiliar program and their levels of transportation and parasocial relationships with favorite characters are compared. Higher levels of transportation and stronger parasocial relationships indicate increased media engagement, a known predictor of increased media effects (Green et al. 2004). Observing a relation between binge watching and narrative engagement would suggest that the changing ways in which audiences are engaging with media content may have significant implications for the strength of media effects on these audiences.

\subsection{Defining Binge Watching}

There is significant debate across both research and industrial communities regarding the definition of binge watching. The Oxford English Dictionary [OED] defines binge viewing as the "practice of watching multiple episodes of a television program in rapid succession, typically by means of DVDs or digital streaming" (OED 2014). A survey of Netflix users (sponsored and commissioned by Netflix) presents a less nebulous, but still broad conceptualization of binge watching: "viewing between two and six episodes of one program in one sitting" (Harris Interactive 2013). Media commentators generally describe binge watching as viewing at least four episodes of a 40-minute program, otherwise viewers are simply watching "a little" television (Feeney 2014). Peterson (2016) identifies several approaches to defining binge watching, from temporal measurements to viewer self-perceptions and state of mind. For the purposes of this research, we define binge watching as the practice of watching multiple episodes, generally three or more, of a television program in rapid succession, a slight specification of the OED definition.

\subsection{The Practice and Impacts of Binge Watching}

Media critics argue that binge watching is the natural result of the technological structure of streaming sites (Feeney 2014). For example, Netflix releases entire seasons of programming at once and when a viewer finishes an episode the Netflix postplay feature automatically begins a countdown to the start of the next episode (Feeney 2014). These technological characteristics are often combined with narrative techniques such as the use of cliffhangers or serial emotional narratives to further encourage binging (Notte 2014). Among media consumers, young people report the highest levels of binge watching, with nearly $70 \%$ of audiences between the ages of 18 and 29 reporting regular binge watching (Matrix 2014).

There are many reasons for the popularity of this practice and binge watching can be intentional and planned or unintentional (Riddle et al. 2017). Motivations for binge watching are varied. According to limited data released by Netflix, viewers' most commonly reported motivation to binge watch was a desire to increase their enjoyment of programming, followed by their impatience to see what happens next (Harris Interactive 2013). Specific to binge watching, cultural commentators have suggested psychological motivations such as stress, relaxation, and the desire to unwind (Feeney 2014). Others have suggested that people binge watch out of a desire for acceptance and a need for cultural commonality (McCracken 2013). Empirical research supports these conclusions, finding that viewers binge watch for hedonic reasons, for relaxation, to pass time, for engagement, and for social utility (Pittman and Sheehan 2015) and also to gain a sense of control (Shim and Kim 2018). It has even been suggested that the affordances provided by binge watching, namely increased consumer agency, might relate to consumer empowerment and broader engagement beyond media (Groshek and Krongard 2016).

Research examining the impacts of binge watching on engagement with media or media effects is limited. Pikul (2014) argues that binge watching has a negative physical effect, causing lethargy due to sitting for prolonged periods of time. Riddle et al. (2017) examined the risks of addiction to binge watching, finding that unintentional binging was associated with addiction symptoms such as increased impulsivity, displacement of other behaviors, and continued viewing. This research also 
suggested the need to further examine how (or if) binge watching changes common media effects such as cultivation and desensitization. The first step to answering this challenge is to examine whether or not binge watching impacts how a viewer experiences and engages with a narrative.

\subsection{Narrative Engagement and Binge Watching}

In the media effects literature, increased engagement with a media narrative is associated with stronger effects of media on consumers (Green et al. 2004). Audiences also report seeking out engaging media (Busselle and Bilandzic 2009). This study seeks to examine whether binge watching impacts media engagement via two common indicators of media engagement: narrative transportation and parasocial relationships. If there is an impact of binge watching on parasocial relationships and narrative transportation, that would indicate that binge watching leads to increased levels of media engagement in audiences. These two concepts are highly related (Moyer-Gusé 2008) and, as such, leads to our first hypothesis.

Hypothesis 1 (H1). Narrative transportation and parasocial relationship strength in response to a specific narrative will be positively correlated.

\subsubsection{Narrative Transportation}

Narrative transportation has been defined as the phenomenological experience of escaping into the world of a narrative (written or audiovisual) (Green and Brock 2000). This state of narrative transportation is desired by viewers and includes a sense of leaving the viewer or reader's world of origin (Green and Brock 2000). Transportation theory suggests that the more a viewer is carried into the reality of media narratives, the higher their levels of enjoyment and engagement (Green et al. 2004). The ability to transport into a narrative is positively impacted by repeated exposure (Green et al. 2008) and negatively impacted by the presence of intervening factors that disrupt the media narratives (Green et al. 2008). Binge watching maximizes exposure to a narrative within a short period of time while also minimizing interruption and other intervening factors. Some viewers have even defined an experience as a binge only when they were transported into a narrative (Peterson 2016). This leads to our second hypothesis.

Hypothesis 2 (H2). Binge watching will produce higher levels of narrative transportation into viewed narratives than will episodic viewing.

\subsubsection{Parasocial Relationships}

Parasocial relationships with media figures, as originally conceptualized by Horton and Wohl (1956), are perceived relationships with media figures that are functionally similar to interpersonal relationships (Giles 2002). These relationships are symbolic in nature and characterized by repeated exposure to the character or media figure, missing the figure when they are gone, perceived friendship, and the perception of an intimate bond with the figure (Boon and Lomore 2001). Parasocial relationships are the natural result of engagement with narrative media and are often entered into through simple exposure (Schiappa et al. 2007). As in interpersonal relationships, speed of disclosure and reduction of uncertainty (Berger and Calabrese 1975) have been shown to increase the strength of these relationships (Rubin and Perse 1989). Binge watching increases both the speed of disclosure and increased degree of familiarity, leading to our final hypothesis:

Hypothesis 3 (H3). Binge watching will produce stronger parasocial relationships with viewed characters than will episodic viewing.

Parasocial relationships are defined as in-depth relationships which persist over time (Schramm and Wirth 2010). It is possible that any change wrought by binge watching could be 
short-lived, due to the compressed nature of viewing. Although the immediate experience of parasocial interaction might be enhanced and concentrated during binge watching, the effect on parasocial relationships might decay over time. As such, we propose a final research question.

RQ1: Are changes in parasocial relationships related to binge watching (if any occur) enduring or fleeting?

If there are significant differences in narrative engagement (as reflected by parasocial relationships and narrative transportation) between binge watching and more traditional approaches to viewing, this would have significant implications for how we might expect narratives viewed in a "binge" to impact and engage audiences, and would set the stage for future lines of research regarding how audiences view media, how that relates to the effect media has on these audiences, and the role of media narratives in our social world.

\section{Results}

In this experiment, we manipulated whether or not participants watched a media narrative on a traditional episodic schedule or on a binge schedule in order to determine the impact of binge watching on media engagement. Based on the definition of binge watching described above, participants in the binge condition watched three episodes of the same show (randomly selected from two shows that had been pilot-tested) within a short time frame; participants in the traditional episodic condition watched one episode per week for three weeks (again, randomly selected from one of the pilot-tested shows). Immediately after viewing, participant levels of parasocial relationships with their favorite characters and transportation into the narrative were measured in an online questionnaire. Our hypotheses were tested by examining results of this questionnaire and comparing levels of media engagement for participants in the binge condition to those in the traditional episodic schedule condition. To answer our research question, one week after watching the final episode and completing the questionnaire, participants were asked to complete another online questionnaire measuring their enduring parasocial relationships with favorite characters.

Participants in this study were undergraduate students at the University of Michigan. A total of 77 students participated. Participants were predominantly women $(76 \%)$ and White $(80 \%, 10 \%$ Asian, $5 \%$ Other, $4 \%$ Black) and came from upper-middle-class households ( $95 \%$ had at least one parent with a Bachelor's degree; $63 \%$ had at least one parent with an advanced degree).

Ninety-one percent of participants had not previously heard of the show that they were randomly assigned to watch (Felicity 1998; Everwood 2002). One participant had seen their assigned show before, and was removed from all further analyses. Approximately half of all participants reported particularly liking their assigned show and 88\% liked the show at least "a little". Hypothesis 1 stated that PSR (parasocial relationship) strength and narrative transportation would be related. Across the full sample, PSR and narrative transportation were correlated at $r=0.61(p<0.01)$. Hypothesis 1 was supported.

As anticipated, there were no significant differences between Felicity and Everwood in awareness, enjoyment, transportation, or parasocial relationships, and so we collapse across show for all remaining analyses which compare the binge (experimental) group (both narratives combined, $n=41$ ) and the episodic (control) group (both narratives combined, $n=36$ ).

Our second hypothesis predicted that participants in the binge condition would report higher levels of narrative transportation immediately after viewing the final episode than participants in the episodic condition. An ANOVA with condition as the grouping variable (binge $=1$ ) showed a significant difference $(F(76)=3.40, p<0.05)$ in narrative transportation across conditions with participants in the binge condition reporting higher levels of transportation $(M=3.18, S D=0.67)$ than participants in the control condition $(M=2.76, S D=0.73, t=2.58, p<0.05)$. Hypothesis 2 was also supported.

Our third hypothesis stated that binge watching would predict parasocial relationship strength with a favorite character. Participants in the binge condition reported significantly stronger parasocial 
relationships with their favorite characters $(M=3.12, S D=0.69)$ than participants in the episodic condition $(M=2.68, S D=0.62, F(76)=3.31, p<0.05)$. Hypothesis 3 was also supported. Figure 1 depicts the effects of condition on PSR and transportation.

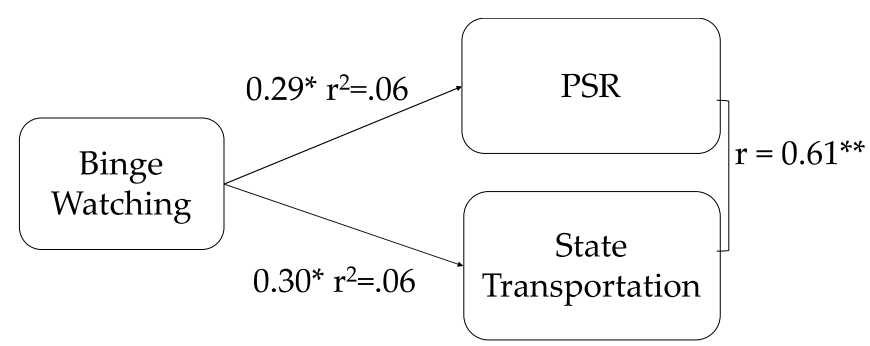

Figure 1. Model of the effect of condition on PSR and state transportation. ${ }^{*} p<0.05{ }^{* *} p<0.01$.

Additionally, a linear regression was employed to determine whether transportation might act as a mediator of binge watching's impact on parasocial relationship strength. Using MacKinnon et al.'s product of coefficients approach (MacKinnon et al. 2002), we found a significant indirect effect of condition on parasocial relationship strength. Figure 2 depicts this mediation model, which results in a nonsignificant relationship between binge watching and PSR and a significant path through transportation. This model accounts for $37 \%$ of variance in PSR.

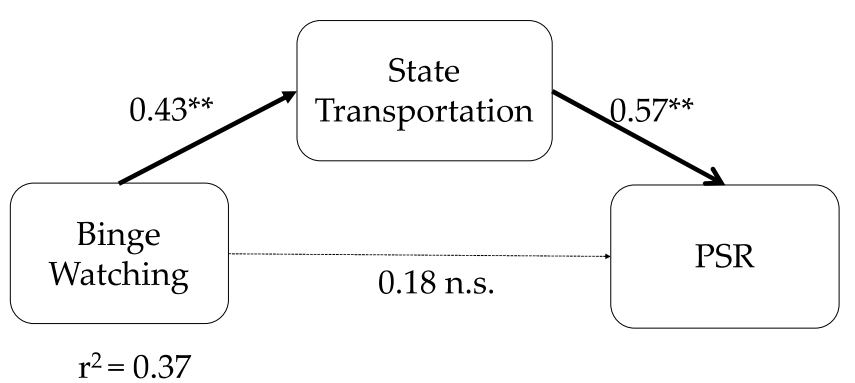

Figure 2. Model of the effect of condition on PSR, mediated by transportation. ${ }^{* *} p<0.01$.

Finally, we asked whether the effect of binge watching on parasocial relationships would endure or decay as time since viewing increased. Results from the follow-up survey measuring parasocial relationships with a favorite character suggest that these effects are enduring. Participants in the binge condition $(M=3.21, S D=0.69)$ had significantly stronger PSRs than those in the nonbinge condition $(M=2.83, S D=0.65)$ one week after viewing $(F(71)=5.60, p<0.05)$.

\section{Discussion}

In this study, we sought to determine whether or not the increasingly common practice of binge watching was associated with changes in audience engagement with media narratives. Using an experimental approach, we manipulated whether or not participants viewed a narrative in a binge-like manner or in a more traditional (one episode per week) manner. All three of our hypotheses were supported. Parasocial relationship strength and narrative transportation, two measures of media engagement, were highly correlated (H1) and binge (compared to episodic) watching predicted both increased transportation (H2) and stronger parasocial relationships with a favorite character (H3). We also asked whether the effects of binge watching would be enduring or short-lived and found evidence for the impact of binge watching on media engagement persisting over time (RQ1).

These findings suggest that the practice of binge watching may have implications for media effects research moving forward. However, the study is not without its limitations. The challenge of manipulating binge watching was to find an ecologically valid approach without sacrificing too much internal validity. By having participants watch their assigned narratives at home, we were able to see 
the results of binge watching in a natural viewing environment, rather than a lab. However, this meant that we had to be somewhat flexible in terms of timing for viewing, giving participants in the binge condition up to three days to view their assigned episodes. This format may not adequately capture the lived practice of binge watching. However, even compressed viewing that approximated binge watching resulted in effects on engagement, suggesting that the actual effect of binge watching may be stronger than reported here. Additionally, participants were assigned a specific narrative to watch via a web-based interface. They did not choose which narrative to watch or when or how to watch that narrative. This contrived, forced aspect of the viewing may have implications for the generalizability of the results. That said, self-selection of media has been shown to increase transportation and engagement (Green et al. 2004); as such, we might expect viewers to be more transported into series that they binge watch by choice than into those they watch in the context of a research study. A final limitation would be the use of a between-subjects approach when analyzing the data collected in the final (follow-up) survey. We asked participants to respond to questions about their parasocial relationships with media figures one week after viewing the final episode. In our study, the participant responses immediately after viewing were not linked to their responses one week later, making a within-subjects analysis comparing time 1 and time 2 impossible. Future examinations of the duration of this phenomenon should use the within-subjects approach.

This study demonstrates that watching multiple sequential episodes of a TV series in a compressed period of time increases audience engagement, at least in the emerging adult population that was studied here (and the age group often cited as cord cutters and binge watchers in popular press). Future research building on these findings could include examination of the effect of self-selection of media and engaging a variety of media audiences. That said, such scholarship must grapple with the fact that what is colloquially referred to as "binge watching" is not a single, new mode of viewing-rather, technological innovations have changed the distribution of content in ways that allow a range of viewing practices. The adoption of the colloquialism by scholars risks oversimplification of nuanced experiences and the conflation of distinct practices and phenomena. We sought to mitigate this somewhat here by precisely and explicitly specifying our definition of binge watching in the current study. This is a necessity, but as research on the topic proliferates and scholars seek to integrate findings across the literature, idiosyncratic and shifting definitions pose challenges to coherent theory building and testing. A coherent and nuanced framework (if not a theory) is needed, as is the development of reliable and valid measures of the phenomena and strategies to test their effects empirically.

At the beginning of this article, we asked whether binge watching (as defined here) engenders deeper connection between audiences and media content. The results of this experiment suggest that it does. These findings highlight the importance of examining more than the content that people are watching when we consider media effects. We need to also examine how audiences are watching and what different viewing practices mean for engagement. Since engagement has been shown to increase media effects (Green et al. 2004), changing viewing practices, like binge watching, that increase engagement have serious implications for how we research and understand media effects. This study provides a first step towards consideration of these implications. Future work should attempt to confirm these suggested implications and to measure their strength. The findings of this study suggest that we may need to consider new approaches to media literacy and to the attenuation of media effects in this changing viewing environment.

\section{Materials and Methods}

This study examined the impact of binge watching on media engagement using an experimental manipulation of viewing style (binge or nonbinge) and measuring parasocial relationships and narrative transportation. Participants were assigned to a binge (three episodes in quick succession) or traditional (one episode per week for three weeks) condition. The traditional condition acted as a control. Immediately after viewing the third episode of their assigned narrative (one of two previously determined by the researchers), participants completed a survey measuring their enjoyment 
of the narrative, their level of narrative transportation, and the presence and strength of a parasocial relationship with their favorite character. To examine the enduring nature of any effects, participants completed a follow-up survey one week after the first survey which measured their parasocial relationships once again.

\subsection{Pilot Testing of Media Narratives}

In order to select the media narratives to use in the experiment, we pilot tested six television programs. Programs were selected based on their targeting of young adult audiences and their dramatic nature, both characteristics expected to increase transportation. We also selected series from around the same time period that would have aired before our participants reached the target demographic. The goal of the pilot test was to find two series that participants enjoyed but were not previously familiar with to use in the experiment. Participants in the pilot test watched the first episode of one of nine series (Brothers and Sisters, Dawson's Creek, Everwood, Felicity, Gilmore Girls, October Road, Roswell, The O.C., and What about Brian?). Ultimately, participants were largely unfamiliar with any of the shows and enjoyed Everwood and Felicity the most, expressing high likelihood of seeking out further episodes of these series. These were the texts utilized in the experiment.

\subsection{Procedure}

Participants were recruited from a large introductory Communication Studies course and received four hours of class credit toward required research participation for their inclusion in the study. Upon signing up for the study, participants were randomly assigned to either the binge condition or the episodic condition and to either Felicity or Everwood, in a 2 by 2 design. In both conditions, participants completed a pre-viewing questionnaire measuring their trait transportability and (immediately after viewing) a post-viewing questionnaire measuring their parasocial relationship with a favorite character from the series they viewed and their state transportation into the narrative. A follow-up questionnaire measuring the enduring parasocial relationship with a favorite character was sent one week after the participant viewed their final episode and completed the post-viewing questionnaire.

In the experimental condition, participants completed the pre-viewing questionnaire and then received a link, via email, to the first three episodes of their assigned series. Participants were given three days from the day the email was sent to watch all three episodes and then complete the post-viewing questionnaire (immediately after viewing the final episode). They were asked to watch all three episodes in one sitting or as close together as possible. After viewing each episode, participants were asked to summarize the plot as an attention check. One week after viewing all three episodes, participants received a link to the follow-up questionnaire.

In the episodic condition, participants completed the pre-viewing questionnaire and then received a link, via email, to the first episode of their assigned series. Upon receiving the link, they had three days to watch the episode. They were asked to watch each full episode in one sitting. One week later, the participants were sent the second episode and one week after the second, the third episode was sent. For each episode, participants had three days to view the episode and were asked to provide a brief summary of the plot (as in the experimental condition). Immediately after viewing the final episode, participants completed the post-viewing questionnaire. One week later, they were sent the follow-up questionnaire.

\subsection{Measures}

\subsubsection{Pre-Viewing Questionnaire}

In the pre-viewing questionnaire, participants completed a trait transportability scale (Dal Cin et al. 2004) which measured the extent to which participants were predisposed, based on their personalities, to being transported into a narrative. Participants were also asked to indicate their gender, race, and parental education levels. 


\subsubsection{Post-Viewing Questionnaire}

The post-viewing questionnaire consisted of three sections. The first section asked for participants' general responses to the assigned narrative. To gauge any previous knowledge of the show or characters, participants were asked if they had ever heard of the show before the study and whether or not they had previously viewed any episodes from their assigned series. They were also asked how much they enjoyed viewing, how likely they were to keep watching the show after the experiment had ended, who their favorite character was, and how attached they felt to the characters in the show.

The second section of the questionnaire measured participants' parasocial relationships with their previously named favorite character. Rubin et al.'s (1985) Parasocial Interaction Scale $(r=0.87)$, which asks participants to agree or disagree (on a five-item Likert scale) with a series of statements about their favorite character such as "I looked forward to watching or listening to this person" and "this person made me feel comfortable, as if I was with friends".

Finally, participants completed Green and Brock's (2000) 10-item Narrative Transportation Scale $(r=0.83)$. For these items, participants were asked to agree or disagree (again on a five-point Likert scale) with statements about their viewing experience. Some of these statements included "While I was watching the show, activity going on in the room around me was on my mind" (reverse coded), "the show affected me emotionally", and "I could picture myself in the scenes of the events of the show".

\subsubsection{Follow-Up Questionnaire}

One week after participants had finished viewing, they were sent a follow-up questionnaire which repeated the Rubin et al. (1985) PSI scale, to determine whether or not any changes in parasocial relationship strength were enduring.

\subsection{Data Analysis}

Data were analyzed using SPSS.

Author Contributions: The three authors contributed in the following ways (Sarah Erickson: S.E., Sonya Dal Cin: S.D., Hannah Byl: H.B.): Conceptualization, S.E., S.D., and H.B.; methodology, S.E., S.D., and H.B.; validation, S.E. and S.D.; formal analysis, S.E.; data curation, S.E.; writing-original draft preparation, S.E.; writing-review and editing, S.E. and S.D.; supervision, S.D.

Funding: This research received no external funding.

Acknowledgments: We would like to acknowledge Kristen Minerva and Sanjay Koduvalli for their assistance in facilitating both the pilot testing and the experiment. Research space was provided by the Howard R. Marsh Center in the Department of Communication Studies at the University of Michigan.

Conflicts of Interest: The authors declare no conflict of interest.

\section{References}

Berger, Charles, and Richard Calabrese. 1975. Some explorations in initation interaction and beyond: Toward a developmental theory of interpersonal communication. Human Communication Research 1: 99-112. [CrossRef]

Boon, Susan D., and Christine D. Lomore. 2001. Admirer-celebrity relationships among young adults. Human Communication Research 27: 432-65. [CrossRef]

Busselle, Rick, and Helena Bilandzic. 2009. Measuring narrative engagement. Media Psychology 4: $321-47$. [CrossRef]

Campbell, Richard, Christopher Martin, and Bettina Fabos. 2012. Television and cable: The power of visual culture. In Media \& Culture: An Introduction to Mass Communication, 8th ed. Boston: Bedford/St. Martins, pp. 143-81.

Dal Cin, Sonya, Mark Zanna, and Geoffrey T. Fong. 2004. Narrative persuasion and overcoming resistance. In Resistance and Persuasion. Edited by Eric S. Knowles and Jay A. Linn. Mahwah: Erlbaum, pp. 175-91.

Everwood, 2002. "Pilot". Directed by Mark Piznarski. Written by Greg Berlanti. The WB, September 16.

Feeney, Nolan. 2014. When, exactly, does watching a lot of Netflix become a 'binge'? The Atlantic 18.

Felicity, 1998. "Pilot". Directed by Matt Reeves. Written by J. J. Abrams and Matt Reeves. The WB, September 29. 
Giles, David. 2002. Parasocial interaction: A review of the literature and a model for future research. Media Psychology 4: 279-305. [CrossRef]

Green, Melanie, and Timothy Brock. 2000. The role of transportation in the persuasiveness of public narratives. Journal of Personality and Social Psychology 79: 701-21. [CrossRef]

Green, Melanie, Timothy Brock, and Geoff Kaufman. 2004. Understanding media enjoyment: The role of transportation into narrative worlds. Communication Theory 14: 311-27. [CrossRef]

Green, Melanie, Sheryl Kass, Jana Carrey, Benjamin Herzig, Ryan Feeney, and John Sabini. 2008. Transportation across media: Repeated exposure to print and film. Media Psychology 11: 512-39. [CrossRef]

Groshek, Jacob, and Sarah Krongard. 2016. Netflix and engage? Implications for streaming television on political participation during the 2016 Presidential campaign. Social Sciences 5: 65. [CrossRef]

Harris Interactive. 2013. Americans taking advantage of ability to watch TV on their own schedules. Harris Interactive, September 8, Press release.

Horton, Donald, and Richard Wohl. 1956. Mass communication and para-social interaction: Observations on intimacy at a distance. Psychiatry 19: 215-29. [CrossRef] [PubMed]

MacKinnon, David, Chondra Lockwood, Jeanne Hoffman, Stephen Sheets, and Virgil West. 2002. A comparison of methods to test mediation and other intervening variable effects. Psychological Methods 7: 83-104. [CrossRef] [PubMed]

Matrix, Sidneyeve. 2014. The Neflix effect: Teens, binge watching, and on-demand digital media trends. Jeunesse 6: 199-38. [CrossRef]

McCracken, Grant. 2013. From Arrested Development to Doctor Who, binge-watching is changing our culture. Wired, May 24.

Moyer-Gusé, Emily. 2008. Toward a theory of entertainment persuasion: Explaining the persuasive effects of entertainment-education messages. Communication Theory 18: 407-25. [CrossRef]

Netflix Annual Report. 2017. Available online: https://www.sec.gov/Archives/edgar/data/1065280/ 000119312511040217 /d10k.htm (accessed on 20 November 2018).

Notte, Jason. 2014. 10 TV Shows to Binge before the Fall Season. Available online: https:/ /www.thestreet.com/ story /12854468/1/10-tv-shows-to-binge-watch-before-the-fall-season.html (accessed on 29 August 2014).

Oxford English Dictionary. 2014. Oxford: Oxford University Press.

Peterson, Theodore. 2016. To binge or not to binge: A qualitative analysis of college students' binge-watching habits. The Florida Communication Journal 44: 77-88.

Pikul, Corrie. 2014. What Your Brain Looks Like after a Netflix Binge. Available online: https://www. huffingtonpost.com/2014/09/04/binge-watching-tv-harmful-to-your-health_n_5732082.html (accessed on 6 December 2017).

Pittman, Matthew, and Kim Sheehan. 2015. Sprinting a media marathon: Uses and gratifications of binge-watching television through Netflix. First Monday 20: 1-11. [CrossRef]

Riddle, Karyn, Alanna Peebles, Catasha Davis, Fangxin Xu, and Elizabeth Schroeder. 2017. The addictive potential of television binge-watching: Comparing intentional and unintentional binge. Psychology of Popular Media Culture 4: 589-604. [CrossRef]

Rubin, Rebecca, and Elizabeth Perse. 1989. Attribution in social and parasocial relationships. Communication Research 16: 59-77. [CrossRef]

Rubin, Alan, Elizabeth Perse, and Robert Powell. 1985. Loneliness, parasocial interaction, and local television news viewing. Human Communication Research 12: 155-80. [CrossRef]

Schiappa, Edward, Mike Allen, and Peter Gregg. 2007. Parasocial relationships and television: A meta-analysis of the effects. In Mass Media Effects Research: Advances through Meta-Analysis. Edited by Raymond W. Preiss, Barbara Mae Gayle, Nancy Burrell, Mike Alan and Jennings Bryant. Mahwah: Erlbaum.

Schramm, Holger, and Werner Wirth. 2010. Testing a universal tool for measuring parasocial interactions across different situations and media. Journal of Media Psychology 22: 26-36. [CrossRef]

Shim, Hongjin, and Ki Joon Kim. 2018. An exploration of the motives for binge-watching and the role of individual differences. Computers in Human Behavior 82: 94-100. [CrossRef]

(C) 2019 by the authors. Licensee MDPI, Basel, Switzerland. This article is an open access article distributed under the terms and conditions of the Creative Commons Attribution (CC BY) license (http:/ / creativecommons.org/licenses/by/4.0/). 